\title{
Data-Informed Decomposition for Localized Uncertainty Quantification of Dynamical Systems
}

\author{
Waad Subber*, Sayan Ghosh, Piyush Pandita, Yiming Zhang and Liping Wang
}

Citation: Subber, W.; Ghosh, S.

Pandita, P.; Zhang, Y.; Wang, L.

Data-Informed Decomposition for Localized Uncertainty Quantification of Dynamical Systems. Vibration 2021, 4, 49-63. https://doi.org/10.3390/ vibration 4010004

Received: 19 November 2020 Accepted: 16 December 2020 Published: 31 December 2020

Publisher's Note: MDPI stays neutral with regard to jurisdictional clai$\mathrm{ms}$ in published maps and institutional affiliations.

Copyright: (C) 2020 by the authors. Licensee MDPI, Basel, Switzerland. This article is an open access article distributed under the terms and conditions of the Creative Commons Attribution (CC BY) license (https:// creativecommons.org/licenses/by/ $4.0 /)$.

Probabilistic Design and Optimization Group, GE Research, 1 Research Circle, Niskayuna, NY 12309, USA; sayan.ghosh1@ge.com (S.G.); piyush.pandita@ge.com (P.P.); yiming.zhang@ge.com (Y.Z.); wangli@ge.com (L.W.) * Correspondence: Waad.Subber@ge.com

\begin{abstract}
Industrial dynamical systems often exhibit multi-scale responses due to material heterogeneity and complex operation conditions. The smallest length-scale of the systems dynamics controls the numerical resolution required to resolve the embedded physics. In practice however, high numerical resolution is only required in a confined region of the domain where fast dynamics or localized material variability is exhibited, whereas a coarser discretization can be sufficient in the rest majority of the domain. Partitioning the complex dynamical system into smaller easier-to-solve problems based on the localized dynamics and material variability can reduce the overall computational cost. The region of interest can be specified based on the localized features of the solution, user interest, and correlation length of the material properties. For problems where a region of interest is not evident, Bayesian inference can provide a feasible solution. In this work, we employ a Bayesian framework to update the prior knowledge of the localized region of interest using measurements of the system response. Once, the region of interest is identified, the localized uncertainty is propagate forward through the computational domain. We demonstrate our framework using numerical experiments on a three-dimensional elastodynamic problem.
\end{abstract}

Keywords: Bayesian inference; uncertainty quantification; dynamical systems; inverse problem; machine learning; system Identification; Gaussian process; polynomial chaos

\section{Introduction}

With the increase in demand for high-performance and highly-efficient systems, the complexity of industrial design and manufacturing processes are increasing proportionally, exposing many opportunities for novel technologies as well as many associated technical challenges. For example, advancement in the design of composite structures allows us to reduce weight, advancement in additive manufacturing enables us to reduce cost. Introducing a new technology typically happens at the lowest level of the systems hierarchy (e.g., at the parts or sub-component levels). Extending new technologies to the system level requires rigorous testing for many years. For example, in the eighties, composite material was only used for limited components of an aircraft (i.e., the wing and tail [1]). Recently, however, about 50\% of the material used in the Boeing 787 Dreamliner are composite material [2]. In the industrial setting, the process of adaptation of a new technology can be accelerated by proper assessment of uncertainty at various aspects of the products life cycle. For example, at the design stage of an aircraft wing rib, it is crucial to consider the effect of uncertainty in the material and operation conditions on the aeroelastic dynamics of the wing [3]. At the manufacturing stage, it is important to consider the impact of material uncertainty on the quality control [4,5]. The maintenance stage requires a holistic assessment of the effect of measurement uncertainty on the static and dynamic responses of the wing during structural health monitoring [6].

Quantifying uncertainty at the system level often requires a physics-based computational model for the entire structure. However, in structures such as an aircraft wing, traditional computational models may become too complex and costly for simulating the 
multi-scale dynamical response due to material heterogeneity at the sub-component level. The effect of the sub-component on the entire structure depends on the size, location and loading conditions of the part. It is therefore, necessary to consider a different level of fidelity for the analysis of the sub-components in order to reduce the cost and complexity of uncertainty quantification. To this end, the concept of localized uncertainty propagation for dynamical systems having multi spatio-temporal scales can be utilized to address such issues [7-9].

In this work, we consider assessing the effect of localized uncertainty in a region of interest within the entire structure. The framework is based on two uncoupled steps: (1) identification of the region of interest, (2) quantifying the effect of localized uncertainty. For structures composed of distinct parts, the localized region of interest for uncertainty propagation can be easily identified. Alternatively, measurement data of the system response can be used to identify the localized region of interest. The Bayesian paradigm integrates computational models and observational data in one framework to update the current state of knowledge [10,11]. Estimating the posterior probability density function in the Bayesian method requires solving the forward model many times, which may become challenging for limited computational budget. This issue is often addressed by building a surrogate model such as a Gaussian Process (GP) regression model [12]. The GP models are non-parametric and Bayesian in nature, and they provide uncertainty bounds on their predictions. Once the region of interest is identified, a Polynomial Chaos (PC) expansion $[13,14]$ is used to propagate the uncertain material properties of the localized region through the entire domain. In contrast to References $[7,8]$ the contributions of this work-(1) The partitioning of the domain is inferred from measurement of the system response, (2) Gaussian process model is used as a surrogate in the Bayesian framework, (3) non-intrusive polynomial chaos approach is used for localized uncertainty propagation. The rest of this work is organized as follows-in Section 2, we provide the problem statement and the associated mathematical formulations. Our numerical demonstrations are provided in Section 3. We provide the conclusions of the current work in Section 4.

\section{Methodology}

In this section, we present the mathematical framework of our approach for datadriven partitioning scheme for localized uncertainty quantification. In particular, in Section 2.1, we introduce the problem statement in the Bayesian setting. For problems where the localized region of interest is not defined explicitly, we rely on measurement data of the system response to infer the localized region of interest using Bayesian framework. The Bayesian framework requires a computational model (the forward problem) to estimate the response of the system for a given set of the input parameters. Consequently, in Section 2.2, we discuss the stochastic elastodynamic problem and its finite element discretization. Estimating the localized region of interest in the Bayesian setting necessitates many solutions to the stochastic elastodynamic problem which can become computationally demanding. A surrogate model for the system response can be used to reduce the computational cost of the Bayesian framework as will be presented in Section 2.3. Once the localized region of interest is estimated, uncertainty representation of the material properties within the region of interest can be performed. The localized uncertainty is propagated forward through the entire computational domain in order to estimate the effect on the material variability on the response. For this task, we use the polynomial chose expansion for efficient assessment of uncertainty with less computational cost. The polynomial chose expansion is reviewed in Section 2.4.

\subsection{Bayesian Inference}

In the Bayesian inference, the prior knowledge is updated to posterior using noisy measurements and the response of a physical model $[10,11]$. The update is based on the Bayes' rule defined as

$$
p(\theta \mid \mathbf{d})=\frac{p(\theta) p(\mathbf{d} \mid \theta)}{p(\mathbf{d})},
$$


where $\theta$ is the unknown parameter to be estimated, $\mathbf{d}$ is the measurement of an observable quantity, $p(\theta \mid \mathbf{d})$ is the posterior probability density function, $p(\theta)$ is the prior probability density function, and $p(\mathbf{d} \mid \theta)$ denotes the likelihood of the observations given the parameter. We assume that the measured data, $\mathbf{d}$, is generated from a statistical model represented as

$$
\mathbf{d}=\mathbb{M}(\theta)+\epsilon,
$$

where $\mathbb{M}(\theta)$ denotes a physical model and $\epsilon$ is a measurement noise represented as a Gaussian random variable with unknown variance $\epsilon \sim \mathcal{N}\left(0, \sigma_{n}^{2}\right)$. For a Gaussian noise, the likelihood function becomes

$$
p(\mathbf{d} \mid \theta)=\frac{1}{\left(2 \pi \sigma_{n}^{2}\right)^{-N / 2}} \exp \left(-\sum_{i}^{N} \frac{\left[d_{i}-\mathbb{M}\left(\theta_{i}\right)\right]^{2}}{2 \sigma_{n}^{2}}\right) .
$$

The task in hand is to utilize the measurement $\mathbf{d}$ and the physical model $\mathbb{M}(\theta)$ to estimate the system parameters $\theta$. The process requires many executions to the physical model $\mathbb{M}(\theta)$, which can be computationally expensive. The computational model is often approximated by a simpler easy to evaluate model as:

$$
\mathbb{M}(\theta) \simeq \mathcal{M}(\theta),
$$

where $\mathcal{M}(\theta)$ denotes the surrogate model that is constructed using limited runs of the physical model $\mathbb{M}(\theta)$. In our work, we represent $\mathcal{M}(\theta)$ as the Gaussian process surrogate model [15]. Once we construct the surrogate model, the localized features parameterized by $\theta$ is estimated using Markov Chain Monte Carlo (MCMC) sampling technique [16,17]. Having identified the region of interest, a localized uncertainty quantification of the material properties can be performed efficiently using polynomial chaos expansion [13].

\subsection{The Forward Problem}

We consider an arbitrary physical domain $\Omega \in \mathbb{R}^{d}$ with $\partial \Omega$ being its boundary as shown in Figure 1a, and define the following problem:

Find a random function $\mathbf{u}(\mathbf{x}, t, \xi): \Omega \times\left[0, T_{f}\right] \times \Xi \rightarrow \mathbb{R}$, such that the following equations hold

$$
\begin{aligned}
& \rho(\xi) \ddot{\mathbf{u}}(\mathbf{x}, t, \xi)=\nabla \cdot \sigma+\mathbf{b} \text { in } \Omega \quad \times\left[0, T_{f}\right] \times \Xi, \\
& \mathbf{u}(\mathbf{x}, t, \boldsymbol{\xi})=\overline{\mathbf{u}} \quad \text { on } \partial \Omega_{u} \times\left[0, T_{f}\right] \times \Xi, \\
& \sigma \cdot \mathbf{n}=\overline{\mathbf{t}} \quad \text { on } \partial \Omega_{t} \times\left[0, T_{f}\right] \times \Xi, \\
& \mathbf{u}(\mathbf{x}, 0, \boldsymbol{\xi})=\mathbf{u}_{0} \quad \text { in } \Omega \times \Xi, \\
& \dot{\mathbf{u}}(\mathbf{x}, 0, \xi)=\dot{\mathbf{u}}_{0} \quad \text { in } \Omega \times \Xi \text {, }
\end{aligned}
$$

where $\rho(\xi)$ is the mass density, $\sigma$ is the stress tensor, $\mathbf{u}$ is the displacement field, $\mathbf{b}$ is the body force per unit volume, $\overline{\mathbf{u}}$ is the prescribed displacement on $\partial \Omega_{u}, \overline{\mathbf{t}}$ is the prescribed traction on $\partial \Omega_{t}, \mathbf{n}$ is a unit normal to the surface, and $\mathbf{u}_{0}$ and $\dot{\mathbf{u}}_{0}$ are the initial displacement and velocity, respectively. Here, we define the stochastic space by $(\Theta, \Sigma, P)$, where $\Theta$ denoting the sample space, $\Sigma$ being the $\sigma$-algebra of $\Theta$, and $P$ representing an appropriate probability measure. The stochastic space is parameterized by a finite set of standardized identically distributed random variables $\xi=\left\{\xi_{i}(\theta)\right\}_{i=1}^{M}$, where $\theta \in \Theta$. The support of the random variables is defined as $\Xi=\Xi_{1} \times \Xi_{2} \times \cdots \Xi_{M} \in \mathbb{R}^{M}$ with a joint probability density function given as $p(\boldsymbol{\xi})=p_{1}\left(\xi_{1}\right) \cdot p_{2}\left(\xi_{2}\right) \cdots p_{M}\left(\xi_{M}\right)$.

For linear isotropic elastic martial, the constitutive relation between the stress and strain tensors is given by:

$$
\sigma=\lambda(\boldsymbol{\xi}) \operatorname{tr}(\varepsilon) \mathbf{I}+2 \mu(\boldsymbol{\xi}) \varepsilon,
$$

where $\lambda(\xi)$ and $\mu(\xi)$ are the Lamé's parameters, I is an identity tensor and $\varepsilon$ is the symmetric strain tensor defined as

$$
\varepsilon=\frac{1}{2}\left(\nabla \mathbf{u}+\nabla \mathbf{u}^{T}\right) .
$$


For a random Young's modulus $E(\mathbf{x}, \xi)$ and deterministic Poisson's ratio $v$, the Lamé's parameters can be expressed as

$$
\lambda(\boldsymbol{\xi})=\frac{E(\mathbf{x}, \boldsymbol{\xi}) v}{(1+v)(1-2 v)}, \quad \mu(\boldsymbol{\xi})=\frac{E(\mathbf{x}, \boldsymbol{\xi})}{2(1+v)} .
$$

We consider the case that uncertainty stems from a localized variability in a confined region within the physical domain. For example as shown in Figure 1b, the variability in the quantity of interest can be attributed to random material properties within the subdomain $\Omega_{2}$. The artificial martial boundaries shown in Figure $1 \mathrm{~b}$ for subdomain $\Omega_{2}$ is estimated using Bayesian inference. Localizing random variability in the neighborhood of the quantity of interest reduces the computational cost of uncertainty propagation in problems where a region of interest can be specified. Depending on the interest in the region, each subdomain can have its local uncertainty representation and the corresponding mesh and time resolution. As a result, the Asynchronous Space-Time Domain Decomposition Method with Localized Uncertainty Quantification (PASTA-DDM-UQ) [7-9] can be utilized. In PASTA-DDM-UQ, spatial, temporal and material decomposition are considered. In this work however, we only consider material decomposition and apply non-intrusive approach for uncertainty propagation.

Consequently, let the physical domain $\Omega$ be partitioned based on the martial variability into $n_{s}$ non-overlapping subdomains $\Omega_{s}, 1 \leqslant s \leqslant n_{s}$ as shown in Figure $1 \mathrm{~b}$ and such that:

$$
\Omega=\bigcup_{s=1}^{n_{s}} \Omega_{s}, \quad \Omega_{s} \bigcap \Omega_{r}=\varnothing \text { for } s \neq r, \quad \Gamma=\bigcup_{s=1}^{n_{S}} \Gamma_{s}, \quad \Gamma_{s}=\partial \Omega_{S} \backslash \partial \Omega .
$$

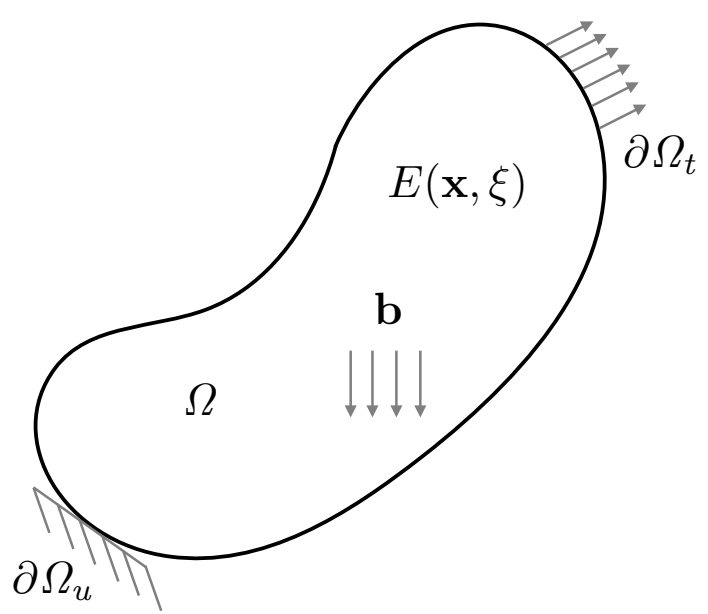

(a) Spatial domain

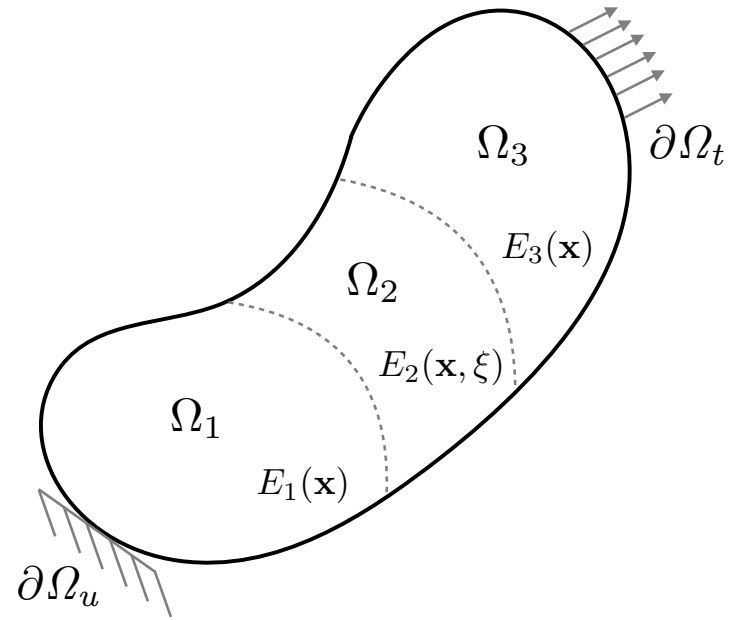

(b) Domain decomposition.

Figure 1. An arbitrary computational domain $\Omega$ with a random material property (i.e., $E(\mathbf{x}, \boldsymbol{\xi}))$ and its partitioning into non-overlapping subdomains. The partitioning is based on material variability.

Note that the partitioning boundaries are not set a priori as it will be estimated using noisy measurement of the system response. According to the decomposition in Equation (9), the stochastic dynamical problem in Equation (5) can be transformed into the following minimization problem:

Find a random function $\mathbf{u}(\mathbf{x}, t, \boldsymbol{\xi}): \Omega \times\left[0, T_{f}\right] \times \Xi \rightarrow \mathbb{R}$, such that

$$
\mathcal{L}(\mathbf{u}, \dot{\mathbf{u}})=\sum_{s=1}^{n_{s}}\left(\mathcal{T}_{s}(\dot{\mathbf{u}})-\mathcal{V}_{s}(\mathbf{u})\right) \rightarrow \min , \quad s=1, \cdots, n_{s}
$$


where $\mathcal{L}(\mathbf{u}, \dot{\mathbf{u}})$ is the Lagrangian of the system, $\mathcal{T}_{s}(\dot{\mathbf{u}})$ denotes the subdomain kinetic energy and $\mathcal{V}_{S}(\mathbf{u})$ is the subdomain potential energy defined as:

$$
\begin{aligned}
& \mathcal{T}_{S}(\dot{\mathbf{u}})=\int_{\Xi} \int_{\Omega_{s}} \frac{1}{2} \rho_{s}(\xi) \dot{\mathbf{u}} \cdot \dot{\mathbf{u}} \mathrm{d} \Omega \mathrm{d} \Xi, \\
& \mathcal{V}_{s}(\mathbf{u})=\int_{\Xi}\left(\int_{\Omega_{S}} \frac{1}{2} \varepsilon: \sigma_{s} \mathrm{~d} \Omega+\int_{\Omega_{S}} \mathbf{u} \cdot \mathbf{b}_{s} \mathrm{~d} \Omega+\int_{\partial \Omega_{t}} \mathbf{u} \cdot \overline{\mathbf{t}}_{s} \mathrm{~d} \Gamma\right) \mathrm{d} \Xi,
\end{aligned}
$$

where $\mathbf{b}_{s}$ and $\overline{\mathbf{t}}_{s}$ are the subdomain body force and the prescribed traction, respectively. The Hamilton's principle with a dissipation term reads

$$
\int_{0}^{T_{f}}\left(\delta \mathcal{L}-\frac{\partial \mathcal{Q}}{\partial \dot{\varepsilon}}: \delta \varepsilon\right) d t=0,
$$

where $\delta \mathcal{L}$ is the first variation of the augmented Lagrangian defined as

$$
\begin{gathered}
\delta \mathcal{L}=\sum_{s=1}^{n_{s}} \int_{\Xi}\left(\int_{\Omega_{s}} \rho_{s}(\xi) \delta \dot{\mathbf{u}} \cdot \dot{\mathbf{u}} \mathrm{d} \Omega-\int_{\Omega_{s}} \delta \boldsymbol{\varepsilon}: \mathbf{D}_{s}(\boldsymbol{\xi}): \boldsymbol{\varepsilon} \mathrm{d} \Omega+\right. \\
\left.\int_{\Omega_{s}} \delta \mathbf{u} \cdot \mathbf{b}_{s} \mathrm{~d} \Omega+\int_{\partial \Omega_{t}} \delta \mathbf{u} \cdot \overline{\mathfrak{t}}_{s} \mathrm{~d} \Gamma\right) \mathrm{d} \Xi
\end{gathered}
$$

here we define $\mathbf{D}_{s}(\boldsymbol{\xi})$ as the uncertain linear elasticity tensor. The dissipation function $\mathcal{Q}(\dot{\mathbf{u}})$ in the Hamilton is defined as

$$
\mathcal{Q}(\dot{\mathbf{u}})=\sum_{s=1}^{n_{s}} \frac{1}{2} \int_{\Xi} \int_{\Omega_{s}} \dot{\varepsilon}: \hat{\mathbf{D}}_{s}: \dot{\varepsilon} \mathrm{d} \Omega \mathrm{d} \Xi, \quad s=1, \cdots, n_{s},
$$

where $\hat{\mathbf{D}}_{s}$ is the damping tensor assumed to be deterministic. Substituting Equations (15) and (14) into the Hamilton's principle Equation (13) gives the following stochastic equation of motion for a typical subdomain $\Omega_{s}$

$$
\begin{gathered}
\int_{\Xi} \int_{\Omega_{s}} \rho_{s}(\xi) \ddot{\mathbf{u}} \cdot \delta \mathbf{u} \mathrm{d} \Omega \mathrm{d} \Xi+\int_{\Xi} \int_{\Omega_{s}} \dot{\varepsilon}: \hat{\mathbf{D}}_{s}: \delta \varepsilon \mathrm{d} \Omega \mathrm{d} \Xi+\int_{\Xi} \int_{\Omega_{s}} \varepsilon: \mathbf{D}_{s}(\boldsymbol{\xi}): \delta \varepsilon \mathrm{d} \Omega \mathrm{d} \Xi \\
=\int_{\Xi} \int_{\Omega_{s}} \delta \mathbf{u} \cdot \mathbf{b}_{s} \mathrm{~d} \Omega \mathrm{d} \Xi+\int_{\Xi} \int_{\partial \Omega_{t}} \delta \mathbf{u} \cdot \overline{\mathbf{t}}_{s} \mathrm{~d} \Gamma \mathrm{d} \Xi .
\end{gathered}
$$

In the next section, we describe the finite element discretization of the weak form defined in Equation (16).

Spatial and Temporal Discretizations

Let the spatial domain $\Omega$ be triangulated with finite elements of size $h$ and let the associated finite element subspace be defined as $\mathcal{X}_{h} \subset H_{0}^{1}(\Omega)$, the spatial component of the solution to the stochastic problem is then sought in the tensor product function space $W=H_{0}^{1}(\Omega) \otimes L^{2}(\Theta)$ defined as $[14,18]$

$$
W=\left\{w(\mathbf{x}, \theta): \Omega \times \Theta \rightarrow \mathbb{R} \mid\|w\|_{W}^{2}<\infty\right\}, \subset H_{0}^{1}(\Omega) \otimes L^{2}(\Theta),
$$

where the energy norm $\|\cdot\|_{W}^{2}$ is defined as

$$
\|w(\mathbf{x}, \theta)\|_{W}^{2}=\int_{\Theta}\left(\int_{\Omega} \kappa(\mathbf{x}, \theta)|\nabla w(\mathbf{x}, \theta)|^{2} \mathrm{~d} \mathbf{x}\right) \mathrm{dP}(\theta) .
$$

The tensor product space $W$ can be viewed as a stochastic space consists of random functions satisfying the Dirichlet boundary condition and having a finite second order 
moment. For a given realization of the underlying random variables of the stochastic space, an approximate finite element solution to the deterministic part can be expressed as

$$
\mathbf{u}^{h}=\sum_{i}^{n_{i}} \mathbf{N}_{i}(\mathbf{x}) \tilde{\mathbf{u}}^{i}(t)
$$

where $\mathbf{N}_{i}(\mathbf{x})$ are traditional spatial finite element basis functions and $\tilde{\mathbf{u}}^{i}(t)$ are the nodal values of the solution as a function of time [19]. Substituting the discrete field, Equation (19) in the weak form Equation (16) gives the following semi-discretized stochastic equation of motion :

$$
\int_{\Xi}(\mathbf{M} \ddot{\mathbf{u}}(t)+\mathbf{C} \dot{\mathbf{u}}(t)+\mathbf{K u}(t)) \mathrm{d} \Xi=\int_{\Xi} \mathbf{F}(t) \mathrm{d} \Xi .
$$

We drop the nodal finite element marks (tilde) for brevity of the representation and define the following matrices:

$$
\begin{array}{ll}
\mathbf{M}=\sum_{s=1}^{n_{s}} \int_{\Omega_{s}} \rho_{s} \mathbf{N}^{T} \mathbf{N} \mathrm{d} \Omega, & \mathbf{C}=\sum_{s=1}^{n_{s}} \int_{\Omega_{s}} \mathbf{B}^{T} \widehat{\mathbf{D}}_{s} \mathbf{B} \mathrm{d} \Omega, \\
\mathbf{K}=\sum_{s=1}^{n_{s}} \int_{\Omega_{s}} \mathbf{B}^{T} \mathbf{D}_{s}^{i} \mathbf{B} \mathrm{d} \Omega, & \mathbf{F}(t)=\sum_{s=1}^{n_{s}}\left(\int_{\Omega_{s}} \mathbf{b}_{s}^{T} \mathbf{N} \mathrm{d} \Omega+\int_{\partial \Omega_{s}} \overline{\mathbf{t}}_{s}^{T} \mathbf{N} \mathrm{d} \Gamma\right) .
\end{array}
$$

Here, $\mathbf{B}$ is the displacement-strain matrix. For time discretization, we use the Newmark time integration scheme to advance the stochastic system one time step as

$$
\begin{aligned}
& \dot{\mathbf{u}}^{k+1}=\dot{\mathbf{u}}^{k}+(1-\gamma) \Delta t \ddot{\mathbf{u}}^{k}+\gamma \Delta t \ddot{\mathbf{u}}^{k+1}, \\
& \mathbf{u}^{k+1}=\mathbf{u}^{k}+\Delta t \dot{\mathbf{u}}^{k}+\left(\frac{1}{2}-\beta\right) \Delta t^{2} \ddot{\mathbf{u}}^{k}+\beta \Delta t^{2} \ddot{\mathbf{u}}^{k+1},
\end{aligned}
$$

where $\gamma$ and $\beta$ are the integration parameters, and $\Delta t=\frac{T_{f}-T_{0}}{n_{t}}$. Substituting he Newmark scheme into the semi-discretized stochastic equation of motion Equation (20), gives the following fully discretized linear system for a give realization of the random vector $\xi$ :

$$
\mathbf{A}(\boldsymbol{\xi}) \mathbf{U}^{k+1}(\boldsymbol{\xi})=\mathbf{F}^{k+1}-\mathbf{G U}^{k}(\boldsymbol{\xi}),
$$

where for compact representation, we define

$$
\begin{array}{ll}
\mathbf{A}(\boldsymbol{\xi})=\left[\begin{array}{ccc}
\mathbf{M}(\mathfrak{\zeta}) & \mathbf{C} & \mathbf{K}(\boldsymbol{\xi}) \\
-\gamma \Delta T \mathbf{I} & \mathbf{I} & \mathbf{0} \\
-\beta \Delta T^{2} \mathbf{I} & \mathbf{0} & \mathbf{I}
\end{array}\right], \quad \mathbf{G}=\left[\begin{array}{ccc}
\mathbf{0} & \mathbf{0} & \mathbf{0} \\
-(1-\gamma) \Delta T \mathbf{I} & -\mathbf{I} & \mathbf{0} \\
-\left(\frac{1}{2}-\beta\right) \Delta T^{2} \mathbf{I} & -\Delta T \mathbf{I} & -\mathbf{I}
\end{array}\right], \\
\mathbf{U}(\boldsymbol{\xi})=\left\{\begin{array}{c}
\ddot{\mathbf{u}}(\boldsymbol{\xi}) \\
\dot{\mathbf{u}}(\boldsymbol{\xi}) \\
\mathbf{u}(\mathcal{\xi})
\end{array}\right\}, \quad \mathbf{F}=\left\{\begin{array}{l}
\mathbf{f} \\
\mathbf{0} \\
\mathbf{0}
\end{array}\right\} . &
\end{array}
$$

For the data-driven decomposition approach, many solutions to the forward problem Equation (23) are required to estimate the appropriate decomposition for localized uncertainty propagation. To mitigate the computational cost involved with identifying the underlying localized region of interest, a Gaussian Process (GP) surrogate model is utilized as explained in the next section.

\subsection{Surrogate Modeling}

The Gaussian Process (GP) surrogate model is widely used for engineering problems as a cost-effective alternative to costly computer simulator [20,21]. In GP for dynamical systems, we consider $\mathcal{D}=\left\{\left(\mathbf{x}_{i}, \mathbf{y}_{i}\right) \mid i=1,2, \cdots, N\right\}$ to be a set of training data consists 
of $N$ samples, where $\mathbf{x}_{i} \in \mathbb{R}^{d}$ represents the input sample $i$, and $\mathbf{y}_{i}$ is the corresponding output vector of size $n_{T}$. For time-series data, the output is observed at a sequence of time steps $t_{j} \in\left[t_{1}, t_{2}, \cdots, t_{n_{T}}\right]$. We concatenate all the input and output into the design matrix $\mathcal{X}$ and the corresponding observation matrix $\mathcal{Y}$, respectively as:

$$
\mathcal{X}=\left[\begin{array}{cc}
t_{1} & \mathbf{x}_{1} \\
\vdots & \vdots \\
t_{n_{T}} & \mathbf{x}_{1} \\
\vdots & \vdots \\
t_{1} & \mathbf{x}_{N} \\
\vdots & \vdots \\
t_{n_{T}} & \mathbf{x}_{N}
\end{array}\right], \quad \mathcal{Y}=\left[\begin{array}{c}
y_{1}^{1} \\
\vdots \\
y_{n_{T}}^{1} \\
\vdots \\
y_{1}^{N} \\
\vdots \\
y_{n_{T}}^{N}
\end{array}\right]
$$

where $y_{j}^{i}$ is the response at time $t_{j}$ for the input parameters $\mathbf{x}_{i}$. The sizes of the design matrix $\mathcal{X}$ and the observation matrix $\mathcal{Y}$ are $\left(N \times n_{T}\right) \times(d+1)$ and $\left(N \times n_{T}\right) \times 1$, respectively. In compact form, the training data set $(\mathcal{X}, Y)$ can be rewritten as:

$$
\mathcal{X}=\left[\begin{array}{ll}
\mathbf{1}_{N} \otimes \mathbf{T} & \mathbf{X} \otimes \mathbf{1}_{n_{T}}
\end{array}\right], \quad \mathcal{Y}=\operatorname{vec}(\mathbf{Y}),
$$

where $\mathbf{1}_{N}$ is an identity vector of size $N, \mathbf{X}=\left[\mathbf{x}_{1}, \cdots, \mathbf{x}_{N}\right]^{T}, \mathbf{T}=\left[t_{1}, \cdots t_{n_{T}}\right]^{T}, \mathbf{1}_{n_{T}}$ is an identity vector of size $n_{T}, \mathbf{Y}=\left[\begin{array}{lll}\mathbf{y}^{1} & \cdots & \mathbf{y}^{N}\end{array}\right]$ and $\mathbf{y}^{i}=\left[y_{1}^{i}, \cdots y_{n_{T}}^{i}\right]^{T}$. Here the symbols $\otimes$ and $\operatorname{vec}(\bullet)$ represent Kronecker product and vectorization operators, respectively. Consequently, a general regression model for time-dependent data can be expressed as a function $f(\mathcal{X})$ that maps the input $\mathcal{X}$ to time-series observation $\mathcal{Y}$. In GP regression, the goal is to infer the function $f(\mathcal{X})$ from noisy observation of the output $\mathcal{Y}$. To this end, the function $f(\mathcal{X})$ is viewed as a random realization of a GP as $f(\mathcal{X}) \sim \mathcal{G P}\left(\mu(\mathcal{X}), \mathbf{K}\left(\mathcal{X}, \mathcal{X}^{\prime}\right)\right)$, where $\mu(\mathcal{X})$ and $\mathbf{K}\left(\mathcal{X}, \mathcal{X}^{\prime}\right)$ are the mean vector and covariance matrix of the process, respectively. Training the GP model can be performed by finding the optimal values to the covariance parameters. Systematically, this is done by maximizing the evidence or the marginal likelihood with respect to the hyperparameter parameters of the kernel. For a zero mean and $\mathbf{K}\left(\mathcal{X}, \mathcal{X}^{\prime}\right)$ covariance matrix, the prediction of the GP based on noisy observation for a new input $\mathbf{x}_{*}$ is a Gaussian process with the following posterior mean and covariance [12]

$$
\begin{aligned}
\mu\left(\mathbf{x}_{*}\right) & =\mathbf{k}\left(\mathbf{x}_{*}, \mathcal{X}\right)\left[\mathbf{K}\left(\mathcal{X}, \mathcal{X}^{\prime}\right)+\sigma_{n}^{2} \mathbf{I}\right]^{-1} \mathcal{Y}, \\
\sigma^{2}\left(\mathbf{x}_{*}\right) & =\mathbf{k}\left(\mathbf{x}_{*}, \mathbf{x}_{*}\right)-\mathbf{k}\left(\mathbf{x}_{*}, \mathcal{X}\right)\left[\mathbf{K}\left(\mathcal{X}, \mathcal{X}^{\prime}\right)+\sigma_{n}^{2} \mathbf{I}\right]^{-1} \mathbf{k}\left(\mathcal{X}, \mathbf{x}_{*}\right) .
\end{aligned}
$$

The covariance function in the GP framework encodes the smoothness and it measures the similarity of the process between two points. The covariance function also encodes the prior belief over the regression function to model the measurements. The prior belief can be on the level of the function smoothness, or behavior and trend such as periodicity. Selecting the right covariance kernel can be challenging for time-dependent data and may require a composition of several covariance functions together to model the right behavior of the data. On the other hand, for problems where the training data is given in the form as in Equation (24), the size of the data may grow exponentially demanding large computational budged. In such a scenario, a scaleable framework for the GP regression of large data set can be exploited to efficiently address the computational cost [22].

In this work, the ultimate goal of the GP model is to serve as a surrogate for the costly simulation code. Thus, we follow a simplified approach to reduce the computational cost of building the surrogate [23]. For the case when the time index of measurement is set a priori and prediction at intermediate time instant is not required, the inter correlation between the time steps can be relaxed. Specifically, the prediction of the model in this case is always set at the location of the measured data, and the model only considers the 
correlation between the input variables $\mathbf{x}_{i}$. Thus the GP can be constructed on the subset of the data $(\mathbf{X}, \mathbf{Y})$ instead of $(\mathcal{X}, Y)$ as $\mathrm{GP}\left(\mu(\mathbf{X}), \mathbf{K}\left(\mathbf{X}, \mathbf{X}^{\prime}\right)\right)$, where

$$
\mathbf{X}=\left[\begin{array}{c}
\mathbf{x}_{1} \\
\vdots \\
\mathbf{x}_{N}
\end{array}\right], \quad \mathbf{Y}=\left[\begin{array}{ccc}
y_{1}^{1}, & \ldots, & y_{n_{T}}^{1} \\
& \vdots & \\
y_{N}^{1}, & \ldots, & y_{n_{T}}^{N} .
\end{array}\right]
$$

\subsection{Polynomial Chaos}

The Polynomial Chaos (PC) expansion is based on the decomposition of a stochastic process into deterministic coefficients scaling random functions. In particular, the PC approximates a stochastic process as a linear combination of stochastic orthogonal basis functions as

$$
\mathbf{u}(t, \boldsymbol{\xi})=\sum_{j=0}^{N} \Psi_{j}(\boldsymbol{\xi}) \mathbf{u}_{j}(t),
$$

where $\Psi_{j}(\boldsymbol{\xi})$ are a set of multivariate orthogonal random polynomials and $\mathbf{u}_{j}(t)$ are the deterministic projection coefficients. The PC coefficients can be estimated non-intrusively as

$$
\mathbf{u}_{j}(t)=\frac{\int_{\Xi} \mathbf{u}(t, \boldsymbol{\xi}) \Psi_{j}(\boldsymbol{\xi}) \mathrm{d} \Xi}{\int_{\Xi} \Psi_{j}^{2}(\boldsymbol{\xi}) \mathrm{d} \Xi}
$$

where $\int_{\Xi}(\bullet) d \Xi$ denotes the expectation operator with respect to the probability density function of the underlying random variables. The expectation integral can be estimated using random sampling or deterministic quadrature rule [24].

\section{Numerical Example}

For the numerical demonstration, we consider the problem of detecting the desired geometry (e.g., localized features) for a given specimen from noisy measurements of its dynamical response. We parameterize the geometry by the dimensions of the inner section (the inner length $l_{i}$ and radius $r_{i}$ ) as shown in Figure 2. The inner dimensions are inferred from noisy measurement of the beam deflection at the mid-span. Once the dimensions are estimated, we perform localized uncertainty propagation of the material parameters of the inner core. For the verification of the framework, a modular approach is considered, where each sub-component of the framework is verified as a standalone unit. This approach is justified by the sequential nature of the coupling mechanism between the building blocks of the framework (i.e., features identification and then uncertainty propagation). This approach quantifies the error in each step and prevents the error measures from being overly influenced by one component compared to another.

\subsection{The Forward Problem}

We consider a 3-D Aluminum beam with mean elastic properties of $E=70 \mathrm{GPa}$, $v=0.3$ and $\rho=26.25 \mathrm{kN} / \mathrm{m}^{3}$. For the damping representation, we consider Rayleigh damping as $\mathbf{C}=\eta_{m} \mathbf{M}+\eta_{k} \mathbf{K}$ with the constants $\eta_{m}=0$ and $\eta_{k}=0.001$. The stiffness $\mathbf{K}$ in the Rayleigh damping is based on the mean properties. We utilize FEniCS [25] for the forward finite element simulations. Figure 2 shows a $2 \mathrm{D}$ projection of the beam geometry, where we parameterize the inner cylinder by length $l_{i}$ and radius $r_{i}$, and the outer cylinder by length $l_{0}$ and radius $r_{0}$. For the reference case, the inner and outer dimensions are $l_{i}=0.45 \mathrm{~m}, r_{i}=0.025 \mathrm{~m}$ and $r_{o}=0.05 \mathrm{~m}$ and, $l_{o}=1.0 \mathrm{~m}$, respectively. The beam is subjected to an impact force defined as: $F(t, \mathbf{x})=\left[0,0, F_{0} t / t_{c} \delta(t-t c)\right]^{T}$, where $F_{0}=-5.0 \mathrm{GN}$ and the ramp time is $t_{c}=0.5 \mathrm{~ms}$. The beam is fixed at both ends and subjected to zero initial displacement and velocity. 


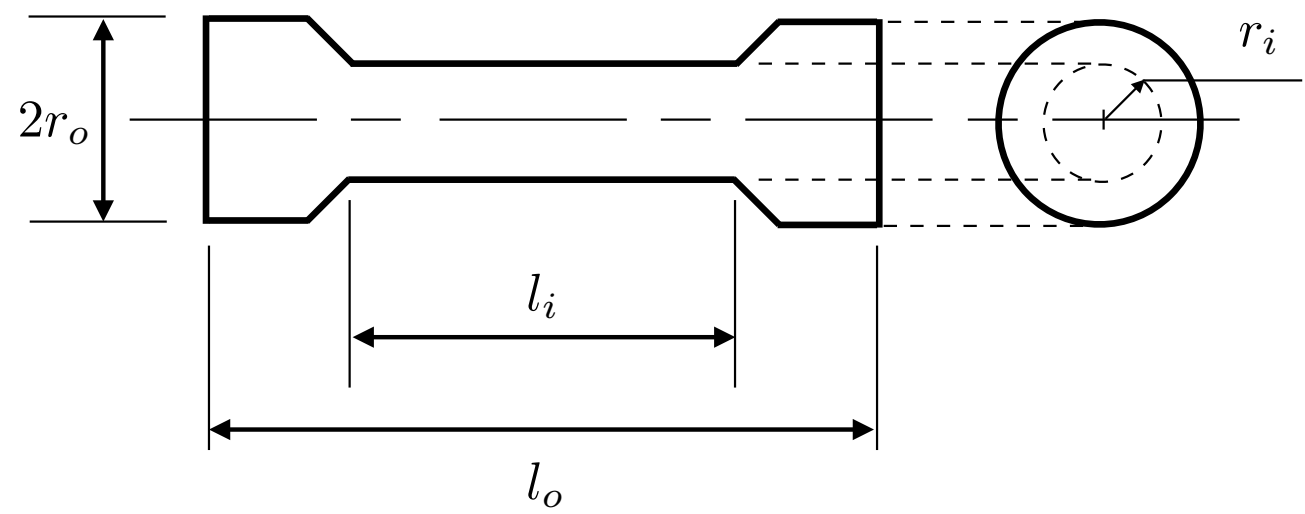

Figure 2. Schematic showing a 2D projection of a typical beam. For the reference case the inner and outer dimensions are $\left(l_{i}=0.45 \mathrm{~m}, r_{i}=0.025 \mathrm{~m}\right)$ and $\left(r_{o}=0.05 \mathrm{~m}\right.$ and, $\left.l_{o}=1.0 \mathrm{~m}\right)$, respectively.

We consider the vertical deflection at the mid-span to be the quantity of interest (QoI) in identifying the underlying beam geometry. Figure 3 shows the mid-span displacement and velocity for a the reference case.

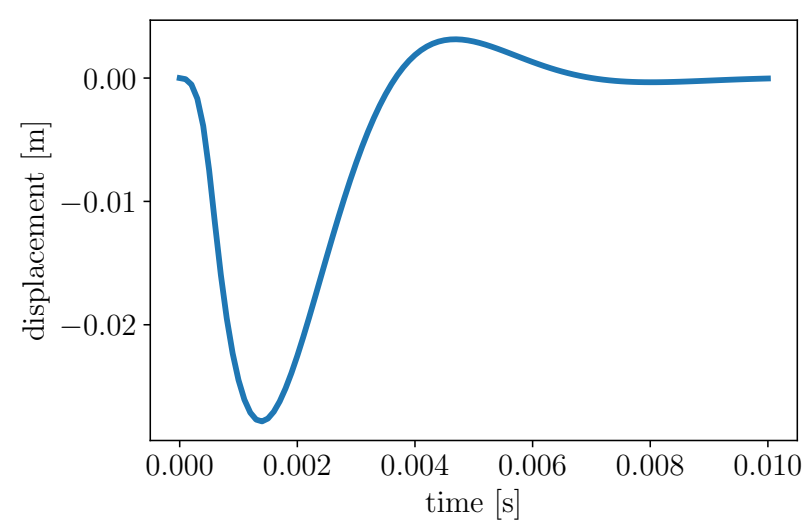

(a) displacement

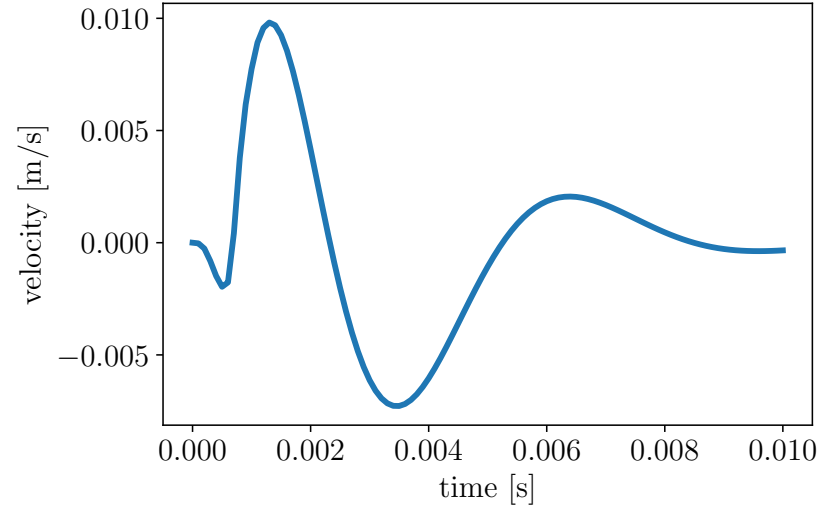

(b) velocity

Figure 3. The displacement and velocity at the mid-span of the reference case for the mean material properties $E=70 \mathrm{GPa}$, $v=0.3$ and $\rho=26.25 \mathrm{kN} / \mathrm{m}^{3}$.

\subsection{The Surrogate Model}

In order to infer the beam geometry from measurement of the QoI, many runs of the forward model, the 3D finite element dynamical code, are required. A surrogate model can overcome this issue by utilizing a limited number of a prespecified runs. The design of computer experiments concept can be used to optimally select the required runs [26-28]. For multi-fidelity simulations, where a high-cost high-accuracy and a low-cost low-accuracy simulators are available, a balance between the computational cost and accuracy can be achieved in designing the numerical simulations experiments [29].

The surrogate model is constructed based on samples that can represent the variability in the beam geometry due to different values of the inner dimensions. We define the variability of the inner dimensions by assigning a uniform random distribution with a specified bounds as $l_{i} \sim U(0.25,0.75) \mathrm{m}$ and $r_{i} \sim U(0.01,0.05) \mathrm{m}$. Using Latin hypercube sampling technique, we generate 50 independent samples for the inner dimensions. Using these samples, we generate the geometry of the beam followed by constructing the corresponding finite element mesh, and executing the forward model to calculate the mid-span deflection (QoI). Samples of the training geometries are shown in Figure 4. Clearly, the samples span a wide range of the probable geometries of the beam. The corresponding scatter of the 
mid-span vertical displacement of the 50 samples is shown in Figure 5. The variability of the inner dimensions not only affect the geometry, but also the location and magnitude of the bouncing deflection at around time $t=0.002 \mathrm{~s}$ and $t=0.005 \mathrm{~s}$.

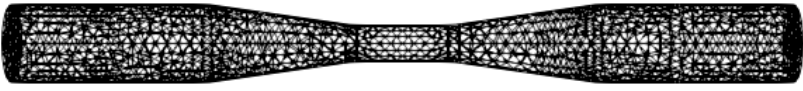

(a) sample 1

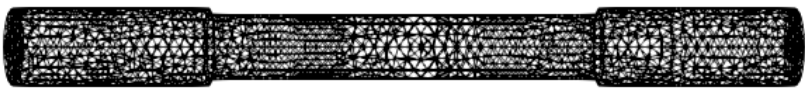

(c) sample 3

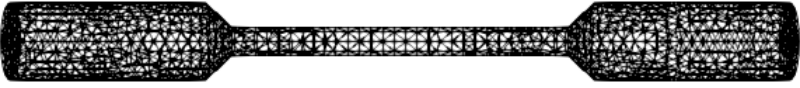

(b) sample 2

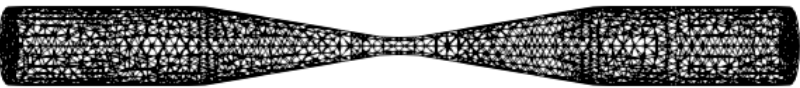

(d) sample 4

Figure 4. Four samples showing the variability in the beam geometry due to different values of the inner dimensions $\left(l_{i}, r_{i}\right)$.

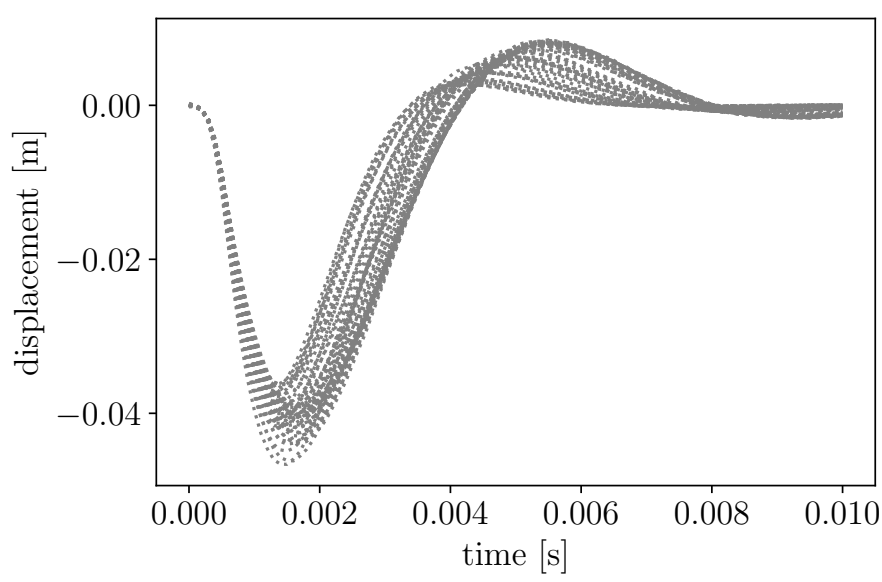

Figure 5. The mid-span vertical displacement of the 50 samples.

We randomly split the 50 samples into two groups as follows-40 samples for training and 10 for testing. For numerical implementation and to mimic the real world, we add a Gaussian random noise of strength (e.g., $10^{-3} \times \max (u)$ ) to the deflection measurements. Figure 6 shows samples of observed and predicted responses with confidence bounds for different values of the inner dimensions. The errorbars (based on two standard deviation) are indistinguishable within the scale of the graph. The maximum and minimum values of the mean squared error between the prediction and the observed response are $2.10 \times 10^{-7}$ and $5.35 \times 10^{-9}$, respectively. Given the fact that the testing samples are not seen by the model during the training phase, the GP model can predict the unseen data within the given accuracy.

To summarize the quality of the prediction, in Figure 7 , we show the $L_{2}$-norm of the observed and predicted QoI. The observed/predicted validation plot indicates that the coefficient of determination between the prediction and observation is $r^{2}=0.98$, and the corresponding mean squared error is $2.53 \times 10^{-6}$. These statistical metrics indicate that the GP model can estimate the unseen geometry from a noisy measurement of the QoI within a given accuracy.

Once the GP model is validated, it can be deployed as a low-cost surrogate for the 3D finite element analysis code. The prediction of GP model takes only a fraction of the time that is needed by the finite element code to estimate the QoI with a fair accuracy. 


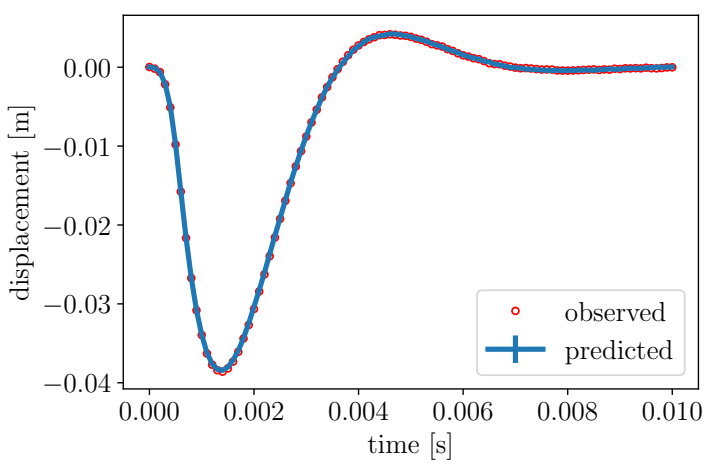

(a) sample 1

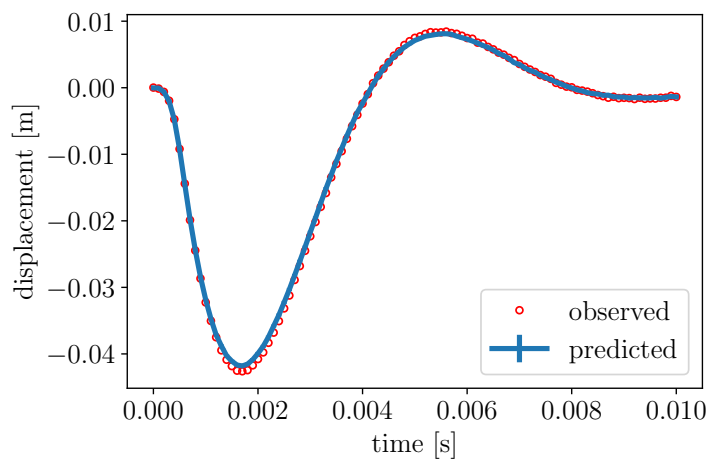

(c) sample 3

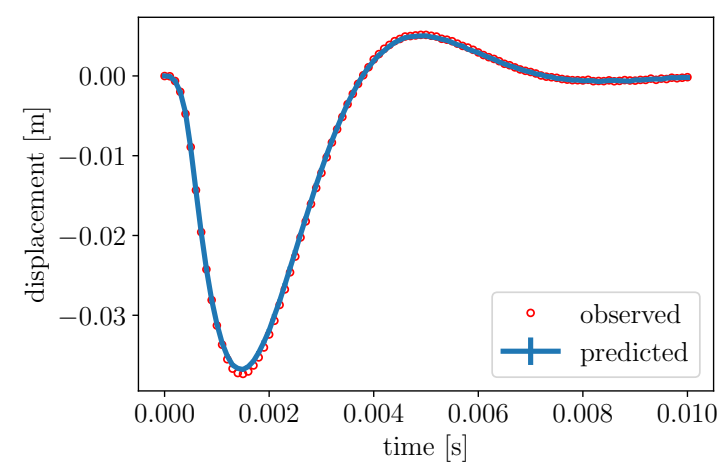

(b) sample 2

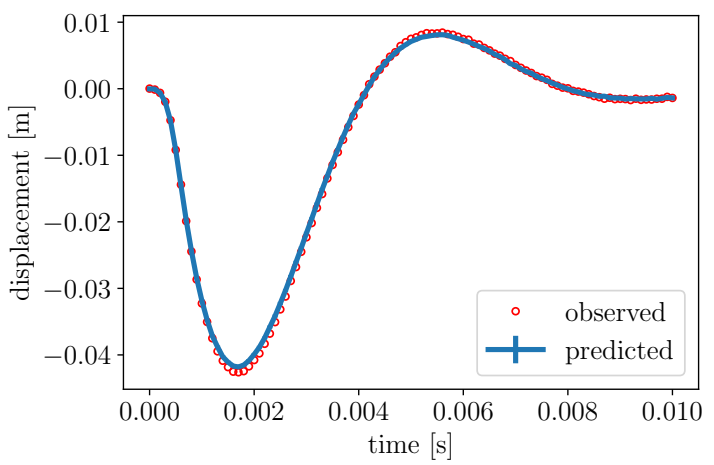

(d) sample 4

Figure 6. Observed and predicted quantity of interest (QoI) for different testing samples. The test samples are not part of the training set. The errorbars are indistinguishable within the scale of the graph.

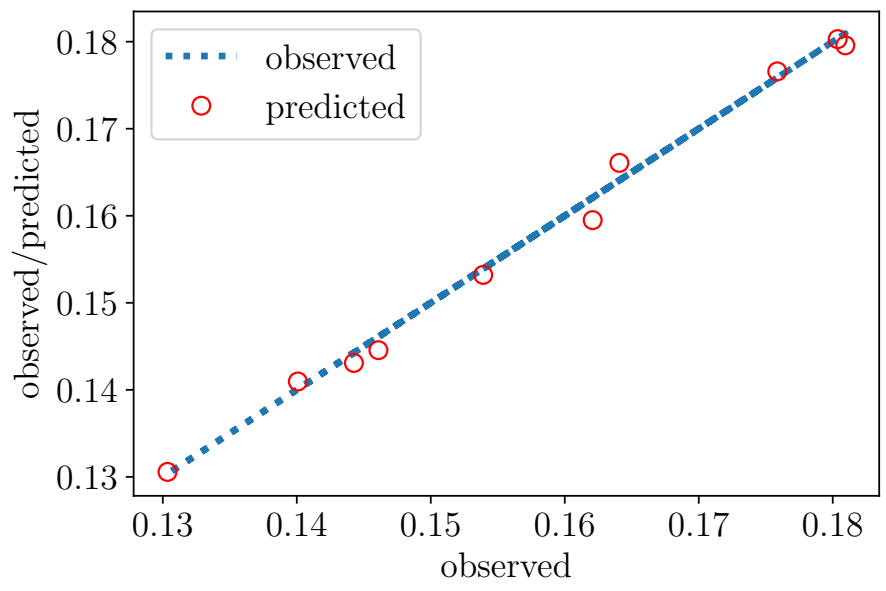

Figure 7. The observed/predicted validation plot showing the norm of the observed (test data) and the corresponding model predictions.

\subsection{The Backward Problem}

In the backward problem, we try to estimate the inner dimensions $\left(l_{i}, r_{i}\right)$ of the beam from noisy measurements of the QoI. We assume that a noisy measurement for the QoI is available as shown in Figure 8. The synthetic data is generated using inner dimension $l_{i}=0.313 \mathrm{~m}$ and $r_{i}=0.055 \mathrm{~m}$ plus $\left(\sigma_{n}=0.1 \times \max (u)\right)$ Gaussian noise to mimic a real experiment setting. 


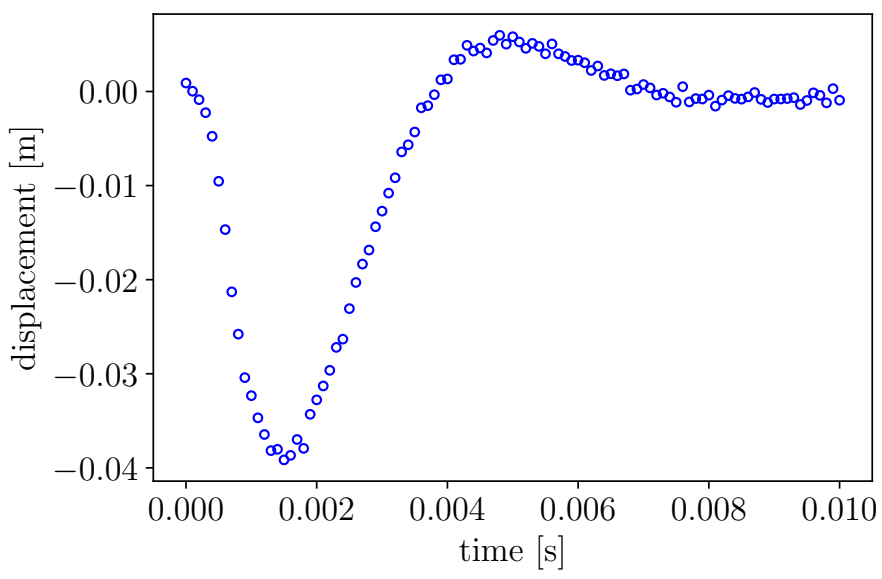

Figure 8. Noisy measurement of the QoI.

For the Bayesian calculation, we use non-informative prior for both the parameters $\theta=\left[l_{i}, r_{i}\right]$ to assess the robustness of the inversion process. An adaptive MCMC method (DRAM) $[16,17]$ is utilized to estimate the posterior density. In Figure 9, we show the estimated posterior density of the parameters $\theta=\left[l_{i}, r_{i}\right]$. We also show the prior density and the true value of the parameters. Note that the true parameters are not part of either the training nor the testing data sets. This highlights the robustness of the framework. The mean of the estimated values are $l_{i}=0.310 \pm 0.048 \mathrm{~m}$ and $r_{i}=0.054 \pm 0.004 \mathrm{~m}$ (the confidence bounds are based on two standard deviation).

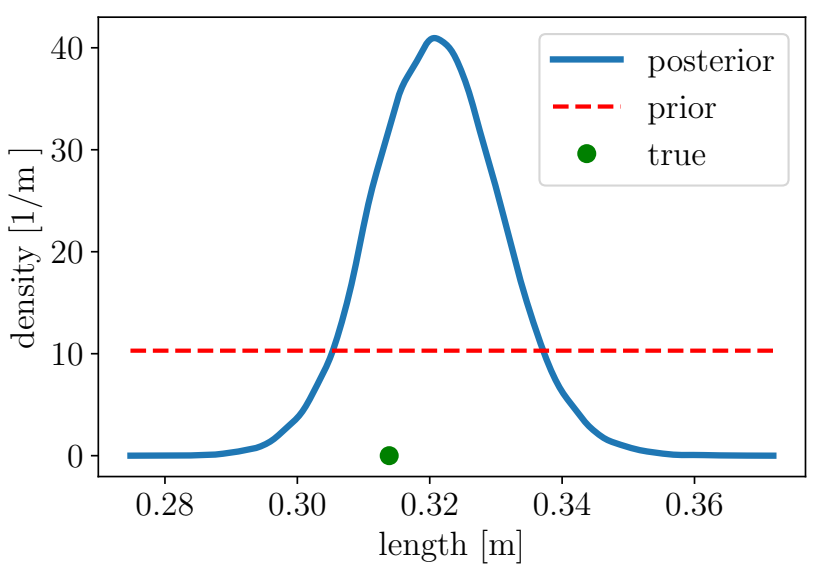

(a) the inner length $l_{i}$

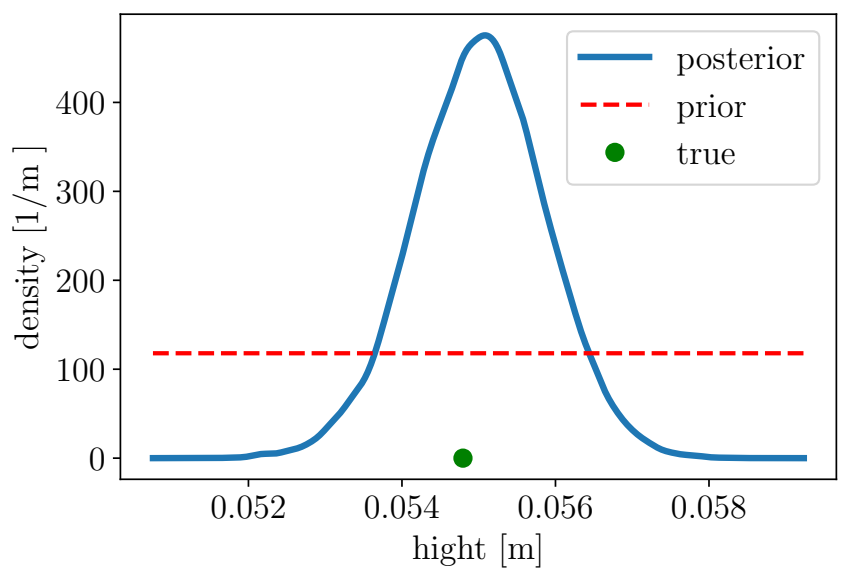

(b) the inner radius $r_{i}$

Figure 9. The estimated posterior density function of the inner dimensions $\theta=\left[l_{i}, r_{i}\right]$. The sold line is the posterior PDF, the dotted line is the prior PDF and the bullet dot represents the true value $l_{i}=0.313 \mathrm{~m}$ and $r_{i}=0.055 \mathrm{~m}$.

Next, the uncertainty in the parameter estimation represented by the posterior density in Figure 9 is propagated forward through the surrogate model to estimate a confidence bounds on the prediction of the QoI. In Figure 10, we show the model prediction and the $95 \%$ confidence interval as well as the true measured response. The $L_{2}$-norm of the discrepancy between the mean model prediction and the measured data is $0.005 \mathrm{~m}$. This conforms that the response due to the estimated parameters uncertainty agrees reasonably well with the true response. Note that, in the estimation of the localized region of interest, the material properties are assumed deterministic. For uncertainty propagation, the Maximum A Posteriori (MAP) estimation is used for the inner dimensions, while assuming random material properties in the region of interest. The relativity small errorbars (within 
the scale of the graph) indicates that the single point estimation MAP can be used to set the inner dimension sufficiently.

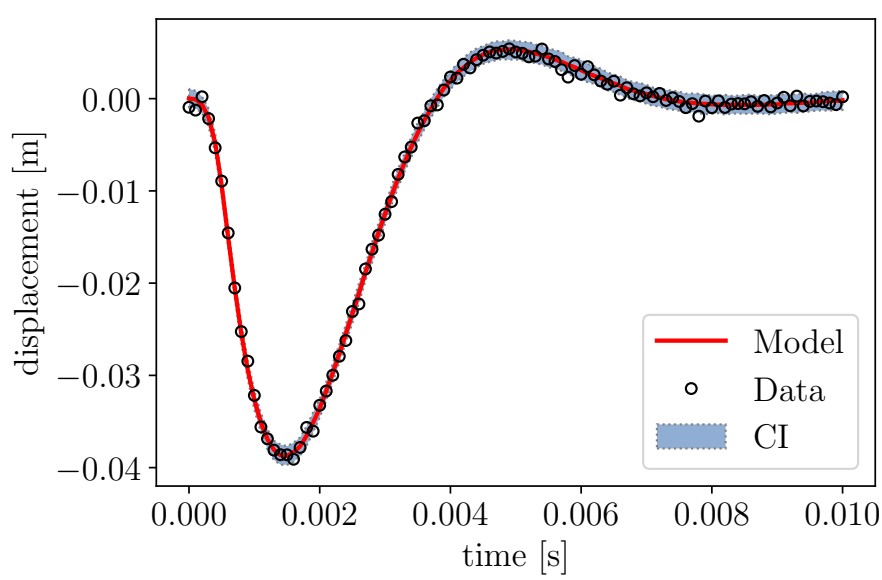

Figure 10. The prediction of the surrogate model and its confidence interval due to uncertainty propagation of the variability in the estimated inner dimensions.

\subsection{Localized Uncertainty Propagation}

The QoI is confined within the core cylinder defined by inner dimensions $\theta=\left[l_{i}, r_{i}\right]$. Once these dimensions are available, the effect of the random variability in the material properties of the inner subdomain can be estimated using PC expansion. Without loss of generality, here we assume that for the inner cylinder, the Young's modulus and material density are random quantities, while Poisson's ratio is deterministic as

$$
E\left(\mathbf{x}, \xi_{1}\right)= \begin{cases}E_{0}\left(1+\sigma_{E} \xi_{1}\right), & \text { for } \mathbf{x} \in \Omega_{2} \\ E_{0}, & \text { otherwise }\end{cases}
$$

and

$$
\rho\left(\mathbf{x}, \xi_{2}\right)= \begin{cases}\rho_{0}\left(1+\sigma_{\rho} \xi_{2}\right), & \text { for } \mathbf{x} \in \Omega_{2} \\ \rho_{0}, & \text { otherwise }\end{cases}
$$

where the artificial boundary for $\Omega_{2}$ are defined by MAP estimation of the inner dimensions $\theta=\left[l_{i}, r_{i}\right], E_{0}=70 \mathrm{GPa}, \rho_{0}=26.25 \mathrm{kN} / \mathrm{m}^{3}, \sigma_{E}=0.25$ and $\sigma_{\rho}=0.15$ and $\xi_{1}, \xi_{2}$ are standard normal random variables. Note that, not only the solution over $\Omega_{2}$ is stochastic, but also over all the whole domain since the spatial finite element and stochastic basis functions are continuous across the domains interfaces. We use second order PC expansion to propagate the localized uncertainty due to the random Young's modulus and material density as shown in Figure 11.

To verify the PCE order, Figure 12 shows the error between the predictions of both the displacement and velocity using second and third order expansion. The error measure is defined as error $(\bullet)=(\bullet)_{3 r d}-(\bullet)_{2 n d}$. The relatively small values of the error confirm that the second order expansion is sufficient for uncertainty propagation for this problem.

The uncertainty bounds follow the trend of the response, with a higher value near the shock location. Although not explored here, high spatio-temporal resolution solver can be directed toward the region of interest, while a less resolution alternative can be assigned to the regions away from the QoI. As demonstrated in References [7-9], PASTA-DDM-UQ approach leads to a customized solver for localized uncertainty propagation with less computational cost. 


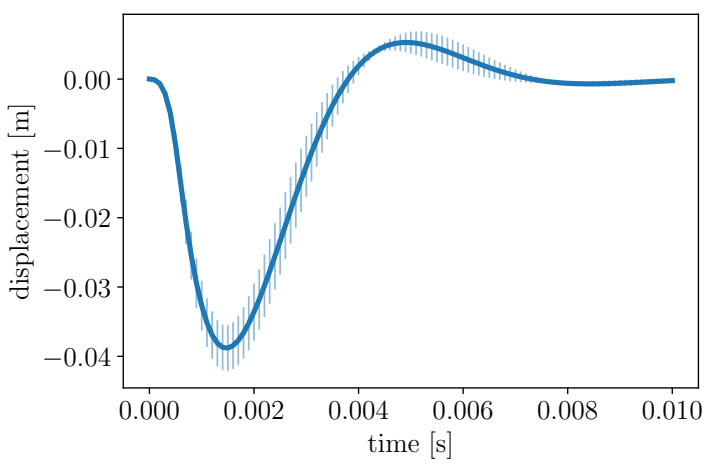

(a) displacement

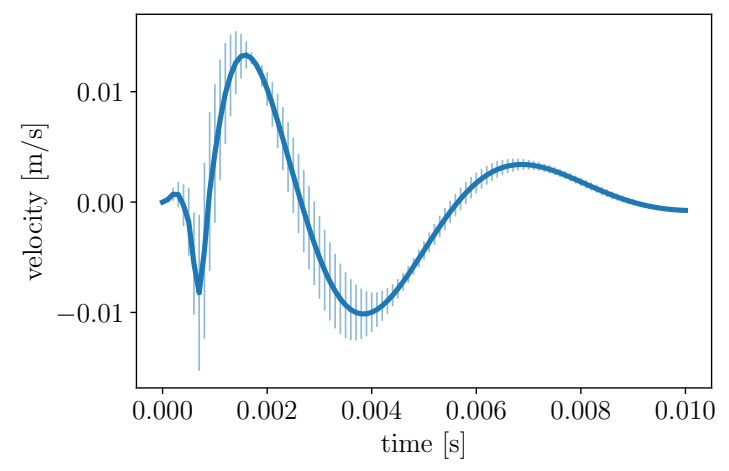

(b) velocity

Figure 11. The Polynomial Chaos (PC) prediction of the displacement and velocity at the mid-span. The uncertainty bounds represent two standard deviation.

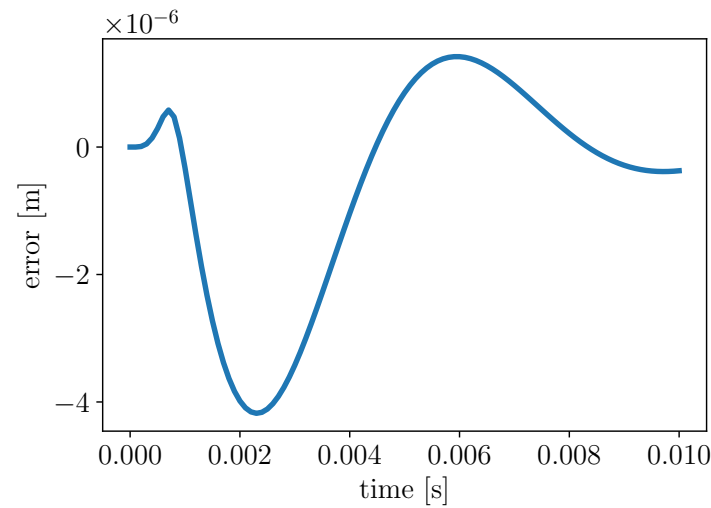

(a) displacement

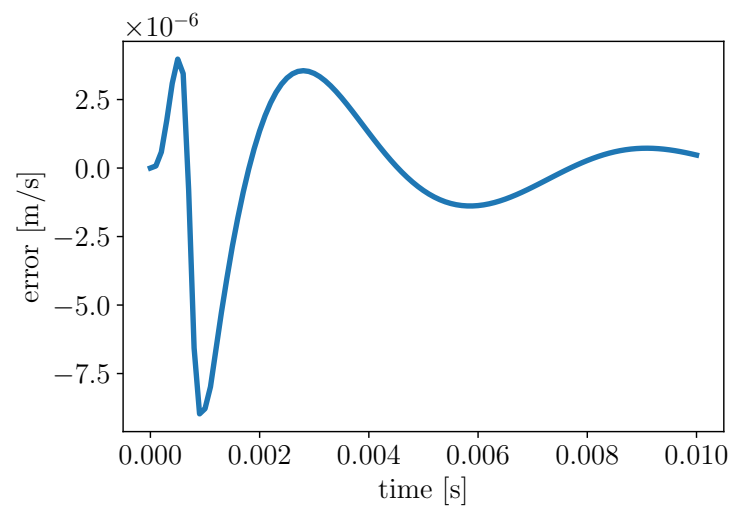

(b) velocity

Figure 12. The error between prediction of the 2nd and 3rd PC order for the displacement and velocity at the mid-span. The error measure is defined as $\operatorname{error}(\bullet)=(\bullet)_{3 r d}-(\bullet)_{2 n d}$.

\section{Conclusions}

We present a data-based partitioning scheme for localized uncertainty quantification in elastodynamic system. The localized region of interest is identified using Bayesian inference framework. Measurement of the system response at one location in conjunction with a physics-based computational model is used to infer the localized features of the region of interested. A data-based surrogate model for the physics-based simulator is constructed using Gaussian process regression in order to reduce the computational cost of the Bayesian inversion. Material uncertainty in the region of interest is propagated through the system using polynomial chaos. We exercise our framework on a three-dimensional beam with localized feature and subjected to an impact load. The presented framework can facilitate quantifying the effect of the confined uncertainty in a localized region of interest within the global computational domain. Proper assessment of uncertainty at various level can accelerate the adaptation process of a new component introduced to an existing system.

Author Contributions: Conceptualization, W.S.; methodology, W.S.; software, W.S.; validation, W.S.; writing—original draft preparation, W.S., S.G., P.P. and Y.Z.; writing—review and editing, W.S., S.G., P.P.; supervision, L.W. All authors have read and agreed to the published version of the manuscript.

Funding: This research received no external funding.

Conflicts of Interest: The authors declare no conflict of interest. 


\section{References}

1. Dutton, S.; Kelly, D.; Baker, A. Composite Materials for Aircraft Structures; American Institute of Aeronautics and Astronautics: Reston, VA, USA, 2004.

2. Mrazova, M. Advanced composite materials of the future in aerospace industry. Incas Bull. 2013, 5, 139.

3. Pettit, C.L. Uncertainty quantification in aeroelasticity: Recent results and research challenges. J. Aircr. 2004, 41, 1217-1229. [CrossRef]

4. Munk, C.L.; Nelson, P.E.; Strand, D.E. Determinant Wing Assembly. U.S. Patent 6,808,143, 26 November 2004.

5. Katunin, A.; Dragan, K.; Dziendzikowski, M. Damage identification in aircraft composite structures: A case study using various non-destructive testing techniques. Compos. Struct. 2015, 127, 1-9. [CrossRef]

6. Diamanti, K.; Soutis, C. Structural health monitoring techniques for aircraft composite structures. Prog. Aerosp. Sci. 2010, 46, 342-352. [CrossRef]

7. Subber, W.; Matouš, K. Asynchronous space-time domain decomposition method with localized uncertainty quantification. Comput. Methods Appl. Mech. Eng. 2017, 325, 369-394. [CrossRef]

8. Subber, W.; Salvadori, A.; Lee, S.; Matouš, K. Uncertainty quantification of the reverse Taylor impact test and localized asynchronous space-time algorithm. In AIP Conference Proceedings, St. Louis, MO, USA, 9-14 July 2017; AIP Publishing LLC: Melville, NY, USA, 2018; Volume 1979, p. 140005.

9. Subber, W.; Matouš, K. Asynchronous space-time algorithm based on a domain decomposition method for structural dynamics problems on non-matching meshes. Comput. Mech. 2016, 57, 211-235. [CrossRef]

10. Smith, R.C. Uncertainty Quantification: Theory, Implementation, and Applications; SIAM: Philadelphia, PA, USA, 2013 ; Volume 12.

11. Gelman, A.; Carlin, J.B.; Stern, H.S.; Rubin, D.B. Bayesian Data Analysis; Chapman \& Hall/CRC: Boca Raton, FL, USA, 2014; Volume 2.

12. Williams, C.K.; Rasmussen, C.E. Gaussian Processes for Machine Learning; MIT Press: Cambridge, MA, USA, 2006 ; Volume 2.

13. Ghanem, R.G.; Spanos, P.D. Stochastic Finite Elements: A Spectral Approach; Courier Corporation: North Chelmsford, MA, USA, 2003.

14. Le Maître, O.; Knio, O.M. Spectral Methods for Uncertainty Quantification: With Applications to Computational Fluid Dynamics; Springer Science \& Business Media: Berlin/Heidelberg, Germany, 2010.

15. Rasmussen, C.E. Gaussian processes in machine learning. In Summer School on Machine Learning; Springer: Berlin/Heidelberg, Germany, 2003; pp. 63-71.

16. Haario, H.; Laine, M.; Mira, A.; Saksman, E. DRAM: Efficient adaptive MCMC. Stat. Comput. 2006, 16, 339-354. [CrossRef]

17. Miles, P.R. pymcmcstat: A Python Package for Bayesian Inference Using Delayed Rejection Adaptive Metropolis. J. Open Source Softw. 2019, 4, 1417. [CrossRef]

18. Babuška, I.; Nobile, F.; Tempone, R. A stochastic collocation method for elliptic partial differential equations with random input data. SIAM J. Numer. Anal. 2007, 45, 1005-1034. [CrossRef]

19. Hughes, T.J. The Finite Element Method: Linear Static and Dynamic Finite Element Analysis; Courier Corporation: North Chelmsford, MA, USA, 2012.

20. Ghosh, S.; Pandita, P.; Subber, W.; Zhang, Y.; Wang, L. Efficient bayesian inverse method using robust gaussian processes for design under uncertainty. In Proceedings of the AIAA Scitech 2020 Forum, Orlando, FL, USA, 6-10 January 2020 ; p. 1877.

21. Ghosh, S.; Pandita, P.; Atkinson, S.; Subber, W.; Zhang, Y.; Kumar, N.C.; Chakrabarti, S.; Wang, L. Advances in Bayesian Probabilistic Modeling for Industrial Applications. ASCE-ASME J. Risk Uncert. Eng. Syst. Part B Mech. Eng. 2020, 6, doi:10.1115/1.4046747. [CrossRef]

22. Zhang, Y.; Ghosh, S.; Pandita, P.; Subber, W.; Khan, G.; Wang, L. Remarks for scaling up a general gaussian process to model large dataset with sub-models. In Proceedings of the AIAA Scitech 2020 Forum, Orlando, FL, USA, 6-10 January 2020 ; p. 0678.

23. Shabouei, M.; Subber, W.; Williams, C.W.; Matouš, K.; Powers, J.M. Chemo-thermal model and gaussian process emulator for combustion synthesis of ni/al composites. Combust. Flame 2019, 207, 153-170. [CrossRef]

24. Feinberg, J.; Langtangen, H.P. Chaospy: An open source tool for designing methods of uncertainty quantification. J. Comput. Sci. 2015, 11, 46-57. [CrossRef]

25. Logg, A.; Mardal, K.A.; Wells, G. Automated Solution of Differential Equations by the Finite Element Method: The FEniCS Book; Springer Science \& Business Media: Berlin/Heidelberg, Germany, 2012; Volume 84.

26. Kristensen, J.; Subber, W.; Zhang, Y.; Ghosh, S.; Kumar, N.C.; Khan, G.; Wang, L. Industrial applications of intelligent adaptive sampling methods for multi-objective optimization. In Design Engineering and Manufacturing; IntechOpen: London, UK, 2019.

27. Kristensen, J.; Bilionis, I.; Zabaras, N. Adaptive simulation selection for the discovery of the ground state line of binary alloys with a limited computational budget. In Recent Progress and Modern Challenges in Applied Mathematics, Modeling and Computational Science; Springer: Berlin/Heidelberg, Germany, 2017; pp. 185-211.

28. Pronzato, L.; Müller, W.G. Design of computer experiments: Space filling and beyond. Stat. Comput. 2012, 22, 681-701. [CrossRef]

29. Ghosh, S.; Kristensen, J.; Zhang, Y.; Subber, W.; Wang, L. A Strategy for Adaptive Sampling of Multi-Fidelity Gaussian Processes to Reduce Predictive Uncertainty. In Proceedings of the International Design Engineering Technical Conferences and Computers and Information in Engineering Conference, Anaheim, CA, USA, 18-21 August 2019; American Society of Mechanical Engineers: New York, NY, USA, 2019; Volume 59193, p. V02BT03A024. 